\title{
Cytocidal Effects of Polyphenolic Compounds, Alone or in Combination with, Anticancer Drugs Against Cancer Cells: Potential Future Application of the Combinatory Therapy
}

\author{
Bo Yuan, Masahiko Imai, Hidetomo Kikuchi, Shin Fukushima, \\ Shingo Hazama, Takenori Akaike, Yuta Yoshino, Kunio Ohyama, \\ Xiaomei Hu, Xiaohua Pei and Hiroo Toyoda
}

Additional information is available at the end of the chapter

http://dx.doi.org/10.5772/50218

\section{Introduction}

A growing body of clinical and experimental evidence has revealed a strong impact of drug resistance on clinical outcomes, especially in cancer therapy, since carcinogenesis is a multistep, multi-pathway and multi-focal process, which involves a series of epigenetic and genetic alterations [1-3]. In order to solve the serious issue facing clinical treatment, combination therapy is now widely advocated for clinical use and has been shown to have a beneficial effect on patient satisfaction [3, 4]. For instance, 5-fluorouracil (5-FU) and leucovorin with either irinotecan or oxaliplatin have been widely used for the treatment of patients with colorectal cancer $[5,6]$. Furthermore, recently, various types of molecular target-based drugs, such as cetuximab and bevacizumab, are being used clinically. Although these continuous efforts to exploit potential combination therapies are ongoing, there is still a growing concern about treatment resistance, disease relapse and side effects of drugs clinically used. Of note, numerous components of edible plants, collectively termed phytochemicals that have beneficial effects for health, are increasingly being reported in the scientific literature and these compounds are now widely recognized as potential therapeutic compounds $[1,2,4,7,8]$. In fact, natural product derived substances, especially polyphenolic compounds with very little toxic effects on normal cells, have attracted great attention in the therapeutic arsenal in clinical oncology due to their chemopreventive, antitumoral, radiosensibilizing and chemosensibilizing activities against various types of aggressive and recurrent cancers [1, 8-10]. 
Apoptosis, or programmed cell death, plays a key role in the development and growth regulation of normal cells, and is often dysregulated in cancer cells [11, 12]. It has been accepted that the aim of anticancer therapy is generally focused on apoptosis induction in premalignant and malignant cells, although other multiple molecular mechanisms such as modulation of carcinogen metabolism, anti-angiogenesis and induction of differentiation are also known to be implicated in its anticancer activity [4, 13]. So far, two principal signal pathways of apoptosis have been identified [11, 12]. The intrinsic mechanism of apoptosis involves a mitochondrial pathway. Apoptosis stimuli destruct mitochondrial membrane structure under the control of Bcl-2 (B-cell leukemia/lymphoma) family, resulting in the release of mitochondrial proteins including cytochrome $c$. Once cytochrome $c$ is released it activates caspase-9 (initiator caspase) through the interaction with Apaf-1 (apoptotic protease activating factor-1) and dATP [14, 15], which ultimately leads to caspase-3 and -7 (effector caspases) activation [16]. On the other hand, the extrinsic pathway induced by death receptors, such as tumor necrosis factor receptor (TNFR) and Fas, is responsible for the activation of caspase- 8 and caspase-10 (initiator caspase) accompanied by the activation of caspase-3 and -7 [16]. The effector caspases are the final mediators in the intrinsic and extrinsic pathways that cleave substrates and lead to cell death. Moreover, a third pathway involving endoplasmic reticulum (ER) stress and caspase-12 has been reported to be associated in apoptosis [4, 13]. A number of markers have been utilized to reveal apoptosis status including cell viability, cytochrome $c$ release, caspase-3 activation, poly (ADP-ribose) polymerase (PARP) cleavage, and DNA fragmentation [17].

Reactive oxygen species (ROS) have been widely believed to play a pivotal role in a wide variety of cellular functions, including cell proliferation and differentiation [3, 11]. Furthermore, oxidative stress, as a result of alterations of redox homeostasis due to an imbalance between ROS production and elimination, is known to be involved in many diseases such as hypoxic injury [11, 18]. Therefore, maintaining ROS homeostasis is crucial for normal cell growth and survival. Generally, cancer cells appear to generate more ROS than do normal cells due to its increased aerobic glycolysis. Furthermore, cancer cells exhibit increased ROS production and altered redox status. Recent studies suggest that these biochemical characteristics of cancer cells can be exploited for therapeutic benefits $[3,18]$. Especially, tumors in advanced stage frequently exhibit multiple genetic alterations and high oxidative stress, suggesting that it is possible to preferentially eliminate these cells by pharmacological ROS insults. However, the upregulation of antioxidant capacity in adaptation to intrinsic oxidative stress in cancer cells can confer drug resistance $[3,18]$. Thus, abrogation of such drugresistant mechanisms by redox modulation could have significant therapeutic implications $[2,3,18,19]$. Indeed, it has been known that altered redox status is closely associated with apoptosis induction in various cancer cells [2, 3, 18, 19]. Collectively, manipulating ROS levels by redox modulation is a way to selectively kill cancer cells without causing significant toxicity to normal cells.

Polyphenolic compounds such as flavonoids and curcumin have been shown to induce apoptosis in various malignant cells including solid tumors and hematologic malignant cells [1, 2, 7-9, 20-22]. Interestingly, the mechanisms underlying the apoptosis induction, 
associated with their antitumoral, chemopreventive and chemotherapeutic activities, have been shown to be implicated in alteration of redox status, since polyphenolic compounds are well known to possess both antioxidant and prooxidant activity [1, 2, 7, 8, 10, 20, 22-26].

In this chapter, we will highlight the recent advances on the cancer preventative activities of the polyphenolic compounds, including flavonoids such as anthocyanins, and Vitex agnuscastus fruit extract (Vitex) in which flavonoids are one of major components, as well as curcumin based on the most recent results from in vitro cell culture and in vivo animal model tumor systems. We will further summarize the detailed mechanisms underlying their cytocidal effects focusing on apoptosis induction. We will also provide detailed insight into potential future clinical application of these promising candidates endowed with potent antitumor activities, alone or in combination with other anticancer clinical drugs based on preclinical and clinical trial results.

\section{Cancer preventative activities of the polyphenolic compounds, anthocyanins, Vitex, and curcumin}

\subsection{Resource and chemistry of anthocyanins, Vitex, and curcumin}

The most abundant flavonoid constituents of fruits and vegetables are anthocyans (i.e. anthocyanins (glycosides), and their aglycones, anthocyanidins) that confer bright red or blue coloration on berries and other fruits and vegetables [8, 20]. Anthocyanins are especially interesting with respect to other flavonoids because they occur in the diet at relatively high concentrations. The daily intake of anthocyanins in the US diet has been suggested to be 180-255 mg/day, in contrast, the daily intake of most other dietary flavonoids, including genistein, quercetin and apigenin, is estimated to be only 20-25 $\mathrm{mg} /$ day [27]. Anthocyanidins are a diphenylpropane-based polyphenolic ring structure, and are limited to a few structure variants including cyanidin, delphinidin, malvidin, pelargonidin, peonidin and petunidin (Figure 1), with a distribution in nature of $50 \%, 12 \%$, $12 \%, 12 \%, 7 \%$, and $7 \%$, respectively, and they present almost exclusively as glycosides, anthocyanins [28]. Furthermore, epidemiological evidence has demonstrated that consumption of fruits and berries has been associated with decreased risk of developing cancer [29].

Vitex agnus-castus is a shrub of the Verbenaceae family and is found naturally in the Middle East and Southern Europe. Ripe fruit of Vitex agnus-castus has been used as a folk medicine for the treatment of various obstetric and gynecological disorders in Europe [30, 31]. Itokawa and colleagues have reported that Vitex (an extract from dried ripe Vitex agnuscastus) possesses cytocidal effects on P388, a mouse leukemia cells, suggesting its antitumor activity, and that flavonoids such as luteolin (Figure 2) are one of its major constituents $[32,33]$.

Curcumin, a hydrophobic polyphenol, also known as turmeric, is a major bioactive ingredient extracted from the rhizome of the plant Curcuma longa [34, 35]. Curcumin has been used as a dietary supplement as well as therapeutic agent in Chinese medicine and 
<smiles>Oc1ccc([O+]=C(c2ccc(O)cc2)c2ccc(O)cc2O)cc1</smiles><smiles>Oc1cc(O)c2cc(O)c(-c3ccc(O)c(O)c3)[o+]c2c1</smiles>

Cyanidin<smiles>OC1=Cc2c(O)cc(O)cc2[C+]=C1c1ccc(O)cc1</smiles>

Pelargonidin<smiles>Oc1cc(O)c2cc(O)c(-c3cc(O)c(O)c(O)c3)[o+]c2c1</smiles>

Delphinidin<smiles></smiles>

Peonidin<smiles></smiles>

Malvidin<smiles></smiles>

Petunidin

Figure 1. Chemical structure of anthocyanidins<smiles>O=c1cc(-c2ccc(O)c(O)c2)oc2cc(O)cc(O)c12</smiles>

Figure 2. Chemical structure of luteolin<smiles>COc1cc(/C=C/C(=O)CC(=O)/C=C/c2ccc(O)c(OC)c2)ccc1O</smiles>

Figure 3. Chemical structures of naturally occurring curcumin. The scheme shows the diketone and keto-enol forms of curcumin. Curcumin exists as an equilibrium mixture of two tautomeric forms in solution. The enol structure of curcumin, which is stabilized by intramolecular H-bonding, is the most energetically stabilized and favored form. 
other Asian medicines for centuries due to its safety and tolerance [34, 35]. Curcumin has a unique conjugated structure including two methylated phenols linked by the enol form of a heptadiene-3,5-diketone that gives the compound a bright yellow color (Figure 3) [7, 35].

\subsection{Involvement of altered redox status in apoptosis induction triggered by polyphenolic compounds}

\subsubsection{Anthocyans}

It has been demonstrated that anthocyanin-rich extracts from berries and grapes, and several pure anthocyanins and anthocyanidins, exhibit pro-apoptotic effects in multiple cell types such as colon [23,36], breast [37, 38], prostate [39, 40], and leukemia cancer cells [10, 41]. They induce apoptosis through both intrinsic (mitochondrial) and extrinsic (Fas) pathways. In the intrinsic pathway, the treatment of cancer cells with anthocyanin results in destabilization of the mitochondrial membrane, cytochrome $c$ release and activation of caspase- 9 , and -3 as well as pro-apoptotic protein such as apoptosis inducing factor $[10,37,40]$. In the extrinsic pathway, anthocyanins modulate the expression of Fas and FasL (Fas ligand) in cancer cells, which result in the activation of caspase-8, then cleaves Bid to tBid, and ultimately stimulates cytochrome $c$ release [41]. Of note, several lines of evidence have indicated that oxidative stress resulted from stimulation of ROS production and/or insufficient ROS elimination is implicated in anthocyanins-triggered apoptosis induction in cancer cells, although broad biological activities including antimutagenesis and anticarcinogenesis of anthocyanins are generally attributed to their antioxidant activity [2, 7, 10, 22-25]. Indeed, it has been demonstrated that the most common type of anthocyanins, cyanidin-3-rutinoside, induced apoptosis in a human myeloid leukemia cell line, HL-60, in a dose- and timedependent manner accompanied by accumulation of peroxides [10]. Cyanidin-3-rutinoside treatment resulted in ROS-dependent activation of p38 mitogen-activated protein kinase (MAPK) and c-Jun N-terminal kinase (JNK), which contributed to cell death by activating the mitochondrial pathway mediated by Bim, one of proapoptotic gene of Bcl-2 family [10]. More importantly, cyanidin-3-rutinoside treatment did not lead to increased ROS accumulation in normal human peripheral blood mononuclear cells (PBMNC) without inducing cytotoxic effects on these cells [10] as indicated by our preliminary data concerning treatment of HL-60 and PBMNC with anthocyanidin (unpublished data). These results suggest that cyanidin-3-rutinoside could be used in leukemia therapy with the advantages of being widely available and selective against tumors. More recently, delphinidin and cyanidin were reported to induce apoptosis in a human metastatic colorectal cancer cell line, LoVo and $\mathrm{LoVo} / \mathrm{ADR}$, a doxorubicin-resistant LoVo, but not in cells originating from a primary tumor site, i.e. Caco-2 [23]. Furthermore, LoVo/ADR was more sensitive to anthocyanins than LoVo cells [23]. It has been reported that the rate of lactate production is significantly higher in LoVo/ADR than in LoVo cells [42]. Therefore, the differences in changes of cellular energy metabolism associated with neoplastic transformation has been suggested to contribute the differential sensitivities to these anthocyanins [43]. Moreover, ROS accumulation, inhibition of glutathione reductase, and depletion of GSH were observed in the apoptosis triggered by anthocyanidins in these metastatic colorectal cancer cell lines [23]. These experimental re- 
sults are in good agreement with previous reports on a possible role of flavonoids, as well as other phytochemicals, in modulating the glutathione (GSH) antioxidant activity, including regulation of intracellular GSH levels through targeting its synthesis, induction of multiple resistant protein 1 mediated GSH efflux, or inhibition of glutathione peroxidase enzyme activity observed in hematopoietic malignant and solid cancer cells in vitro and in vivo [19, $24,44]$. Furthermore, an in vitro study showed that anthocyanidins inhibit glutathione reductase (GR) in an oxygen-dependent manner, presumably via the effect of superoxide [45]. Therefore, these results suggest that anthocyanidins may be used as sensitizing agents through modulating intracellular redox status in various cancer therapy.

Intriguingly, a good correlation has been found between anthocyanin chemical structure and chemoprotective activity. Indeed, several lines of evidence have shown that the number of hydroxyl groups on the B-ring of anthocyanidins is associated with the potency of prooxidative [45-47], apoptotic induction [48], anti-transformation [49], as well as antioxidative activities [1, 8]. For instance, delphinidin and cyanidin that possess orthodihydroxyphenyl structure on the B-ring, showed stronger apoptotic induction in human leukemia cells [48] and inhibitory effect on 12-O-tetradecanoylphorbol-13-acetate (TPA)induced cell transformation [49]. Furthermore, a similar trend of structure-activity relationship was also observed in the suppression of transcription factors closely associated with intracellular redox status, such as activator protein-1 (AP-1), nuclear factor- $\kappa \mathrm{B}$ (NF$\kappa B)$, and CCAAT/enhancer-binding protein (C/EBPS) $[20,49,50]$. Structure-activity studies also suggested that the potency as inhibitors of epidermal growth factor receptor (EGFR), a target of an expanding class of anticancer therapies [51], might be positively correlated with the presence of hydroxyl functions in positions $3^{\prime}$ and $5^{\prime}$ of ring $B$ of the anthocyanidinos molecule, and inversely with the presence of methoxy groups in these positions. All of these findings provide important molecular basis for the antitumor properties of anthocyanidins.

\subsubsection{Vitex}

We have been interesting the effects of naturally derived flavonoids on the growth of various types of cancer cells. Of those, we have demonstrated that Vitex exhibits cytotoxic activities against various types of solid tumor cells, such as KATO-III (a human gastric signet ring carcinoma cell line), COLO 201 (a human colon adenocarcinoma cell line), MCF-7 (a human breast carcinoma cell line) [52]. More interestingly, no apparent cytotoxicity was observed in non-tumor cells, such as human uterine cervical canal fibroblast (HCF) and human embryo fibroblast (HE-21) when treated with concentrations showing significant cytotoxicity in tumor cells, suggesting a selective cytotoxic activity against tumor cells [52]. We further demonstrated that Vitex induced apoptosis accompanied by an accumulation of intracellular ROS along with the decrease in the levels of intracellular GSH in KATO-III cells [22]. At the same time, our experimental data demonstrated a decrease in the amount of Bcl2, Bcl-xL and Bid proteins; an increase in Bad protein; activation of caspase- $8,-9$ and -3 ; a leakage of cytochrome $c$ from mitochondria in the cells [22]. Furthermore, the addition of an antioxidant, N-acetyl-L-cysteine (NAC), or exogenous GSH significantly abrogated the 
effects of Vitex [22]. Together, our results suggest that a crosstalk between intrinsic and extrinsic pathway via Bid activation as a result of oxidative stress plays a critical role in Vitex-induced apoptosis in KATO-III cells. We also demonstrated that apoptosis induction was observed in Vitex-treated COLO 201, concomitantly with a significant increase in heme oxygenase-1 (HO-1) gene expression as observed in KATO-III cells [53]. On the other hand, unlike KATO-III, apoptosis induction was not abrogated in the presence of antioxidants, such as NAC [53]. We further demonstrated that after treatment with Vitex, the upregulation of ER stress-related genes, such as glucose-regulated protein 78 (GRP78) and C/EBPhomologous protein (CHOP) along with the activation of caspase- 9 and -3 were observed in COLO 201 [21]. However, an inhibitor for JNK significantly suppressed the apoptosis induction associated with caspase-3 activation [21]. These results thus suggest that the activation of JNK, and caspase- 9 and -3 resulted from ER stress contributed to the apoptosis induction in Vitex-treated COLO 201 cells. Taken together, it seems that either oxidative stressdependent or ER stress-dependent apoptosis would be triggered in cancer cells treated with Vitex depending on different cell types. Most importantly, our in vivo experimental data revealed that the administration of Vitex significantly suppressed tumor growth in COLO 201 xenograft mice, although more studies must be conducted to understand detailed in vivo pharmacological characterization of Vitex treatment [21]. In addition, as shown in Figure 4, we recently demonstrated a significant dose-dependent cytocidal effect in both a welldifferentiated hepatocellular carcinoma (HCC) cell line, HepG2 and an undifferentiated HCC cell line, HLE, although the levels of cytotoxic activities of Vitex varied between two cells. Similarly, a significant dose-dependent cytocidal effect was also observed in both cells when treated with as high as $20 \mu \mathrm{g} / \mathrm{ml}$ luteolin, one of major constituents of Vitex. However, there was a trend to increase cell proliferation in both cells when treated with a relatively lower concentration of luteolin. On the other hand, 5-FU induced a dose-dependent cytocidal effect on HLE, but not in HepG2. These results indicated that while the cytocidal effect of 5-FU was more selective against undifferentiated hepatocellular carcinoma HLE, Vitex and luteolin exhibited significant cytocidal effects on both well-differentiated and undifferentiated hepatocellular carcinoma cells, suggesting a possible broad usefulness of these compounds to hepatocellular carcinoma therapy. Of note, Vitex has been used to treat patients with various obstetric and gynecological disorders in Europe [30, 31]. Moreover, it is interesting to note that Vitexins, which are isolated from the seed of Chinese herb Vitex Negundo and bear a basic flavonoid structure, show cytotoxic and antitumor effects against breast, prostate and ovarian cancer cells through apoptosis induction via an intrinsic pathway based on in vitro and in vivo xenograft tumor models [54]. Therefore, our results provide a new insight into the clinical use of Vitex for colon cancer and hepatocellular carcinoma besides those cancers mentioned above.

\subsubsection{Curcumin}

Curcumin has emerged worldwide as a potent therapeutic substance for treating diverse human diseases including various types of cancer, such as leukemia, colon cancer and pancreatic cancer $[7,25,35,55,56]$. Although the precise mode of action of this compound is 

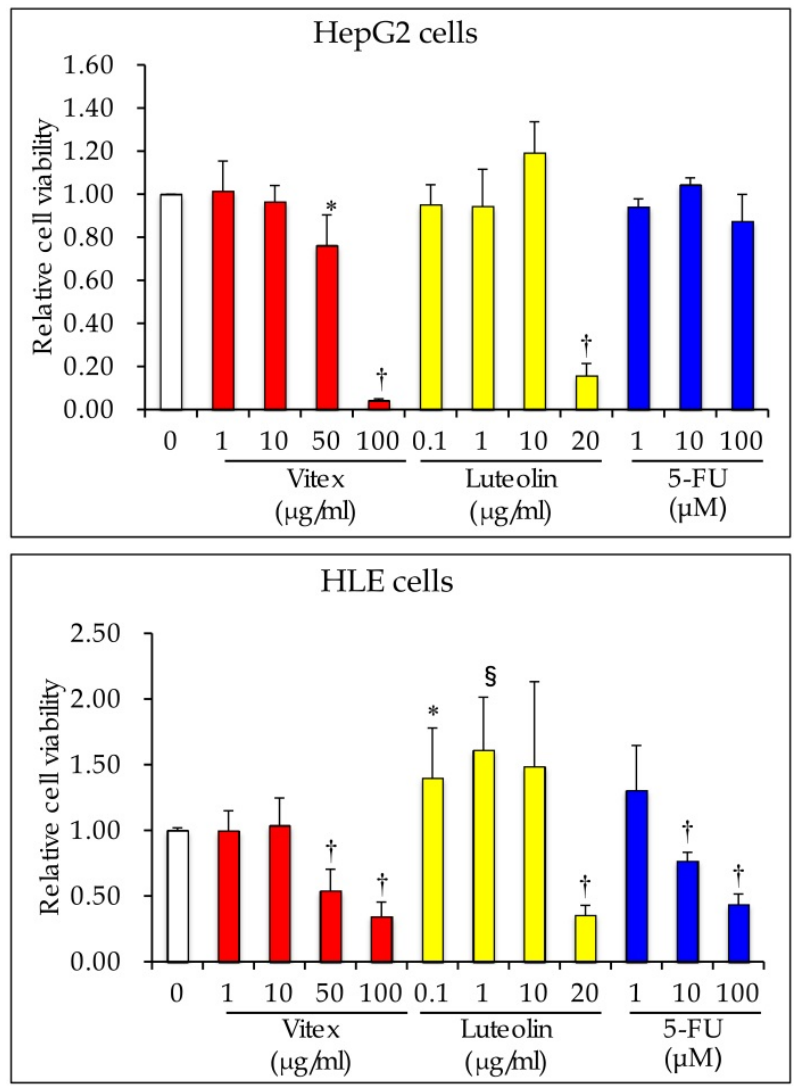

Figure 4. Cytocidal effects of Vitex, luteolin and 5-FU on HepG2 and HLE cells. Hepatocellular carcinoma (HCC) cell lines, HepG2 (well-differentiated) and HLE (undifferentiated) were kindly provided by Dr. Yamato Kikkawa (Laboratory of Clinical Biochemistry, School of Pharmacy, Tokyo University of Pharmacy and Life Sciences, Tokyo, Japan). After treatment with Vitex (final concentrations: 1, 10, 50 and $100 \mu \mathrm{g} / \mathrm{ml}$ ), luteolin (final concentrations: $0.1,1,10$ and $20 \mu \mathrm{g} / \mathrm{ml}$ ) or 5-FU $(1,10$ and $100 \mu \mathrm{M}$ ) for 48 $\mathrm{h}$, cell viability was determined by XTT dye-reduction assay according to the method described previously [53]. Significant differences between treatment group and control $\left({ }^{*}: p<0.05 ; \S: p<0.01 ;+: p<0.001\right)$

not yet elucidated, studies have shown that chemopreventive action of curcumin might be due to its ability to induce apoptosis through multiple signaling pathways, including intrinsic and extrinsic pathways as well as ER stress pathway $[7,57]$. It has been suggested that curcumin-induced apoptosis is associated with ROS production and/or oxidative stress in cancer cells, in spite of its normal antioxidant capacity [7, 57]. Indeed, it has been demonstrated that curcumin can generate ROS as a prooxidant in the presence of copper in HL-60 cells, resulting in DNA damages and apoptotic cell death [58]. Furthermore, the prooxidant action of curcumin may be related to the conjugated $\beta$-diketone structure of this compound [58]. Kuo et al. also demonstrated that curcumin induced a dose- and timedependent apoptotic cell death in the same cells, concomitant with a decrease of Bcl-2 
expression [59]. However, the antioxidants, NAC, L-ascorbic acid, alpha-tocopherol, catalase and superoxide dismutase, all effectively prevented curcumin-induced apoptosis, suggesting that curcumin-mediated apoptosis was closely related to the increase in intracellular ROS [59]. Besides hematopoietic cancer cells, curcumin-mediated apoptosis in human breast epithelial cells (H-ras MCF10A) involved generation of ROS as well as downregulation of Bcl-2 and up-regulation of Bax, suggesting redox signaling as a mechanism responsible for curcumin-induced apoptosis in these cells [60]. Syng-Ai et al. also demonstrated that curcumin-induced apoptosis in human breast tumor cell lines (MCF-7, MDAMB) and HepG2 cells is also mediated through the generation of ROS, and that depletion of glutathione by buthionine sulfoximine (BSO) promoted the increased generation of ROS, thereby further sensitizing the cells to curcumin [61]. Interestingly, curcumin had no cytocidal effect on normal rat hepatocytes, because of no superoxide generation [61]. These observations suggest that curcumin with broad biological actions could be developed into an effective chemopreventive and chemotherapeutic agent based on its ability to modulate intracellular redox status. However, the use of curcumin as a therapeutic agent has met with considerable skepticism, since as much as $75 \%$ of curcumin is excreted in the feces [62] and also undergoes repaid inactivation by glucuronidation [63], similar to metabolisms of flavonoids [2]. Recently, in order to increase its metabolic stability, numerous approaches have been undertaken, such as generating the fluoro-analog of curcumin termed Diflourinated-Curcumin (referred to as CDF) that exhibits increased metabolic stability [64, 65]. Furthermore, the CDF has been found to exhibit superior growth inhibitory properties against cancer cells to the parental compound curcumin $[56,64,65]$.

\section{Potential future application of polyphenolic compounds, alone or in combination with, anticancer drugs}

As mentioned in the previous section, the deregulation and sustained activation of multiple tumorigenic pathways are typically implicated in cancer development and progression with locally advanced and aggressive nature. Consequently, the use of therapeutic agents acting on different deregulated gene products, alone or in combination therapy, may represent a potentially better strategy than the targeting on one specific oncogenic product to overcome treatment resistance and prevent cancer development and disease recurrence [2-4, 7]. So far, one of the most successful models for combinatory cancer therapies is all-trans retinoic acid (ATRA)/arsenic trioxide (ATO, arsenite) combination as a synergistic therapy for acute promyelocytic leukemia (APL) patients, in which ATRA synergizes ATO activity to provide a superior efficacy of combination therapy in patient through promoting the effects of ATO on several signaling pathway, such as apoptosis induction, differentiation as well as the degradation of PML-RAR $\alpha$ [a fusion gene between promyelocytic leukemia (PML) gene and retinoic acid receptor (RAR) $\alpha$ ], a causative gene for APL $[4,13]$. In order to understand the mode of action of ATO and provide an effective treatment protocol for individual APL patients, we recently conducted studies on the pharmacokinetics of ATO in APL patients using biological samples such as peripheral blood (PB) and cerebrospinal fluid, and demonstrated that not only inorganic arsenic but also methylated arsenic metabolites 
accumulated in red blood cells during the consecutive administration of ATO to APL patients [66]. Furthermore, we have demonstrated for the first time that these arsenic metabolites also existed in cerebrospinal fluid [67], in which the concentrations of arsenic reached levels necessary for differentiation induction $[68,69]$. We further investigated for the first time the arsenic speciation in plasma of bone marrow (BM), and demonstrated that speciation profiles of BM plasma were very similar to those of PB plasma, suggesting that speciation analysis of $\mathrm{PB}$ plasma could be predicative for BM speciation [70]. These findings on the pharmacokinetics of ATO in APL patients provide a new insight into clinical applications of ATO, and may contribute to better therapeutic protocols [4]. Recently, in order to understand the clinical side effects of arsenite, we also investigated the effects of arsenite on human-derived normal cells, since the clinical side effects of drugs are always found as a harmful and undesired effect on normal cells and/or tissues. Based on a study using a unique in vitro cell culture system comprising the primary culture chorion and amnion cells established in our laboratory [71-73], we demonstrated for the first time that transporter genes, such as aquaporin 9 and multidrug resistance associated protein 2 are involved in controlling intracellular arsenic accumulation in these primary cultured normal cells, which then contribute to differential sensitivity to arsenite cytotoxicity among these cells [74].

The successful clinical efficacy of ATO in the treatment of APL patients has led to investigations on exploring potential treatment applications for other malignancies, including ATO-resistant hematopoietic cancer and solid tumors [75, 76]. In order to further extend our previous study and promote the clinical application of arsenite, we have been seeking to explore potential candidate agents, which are expected to not only potentiate the efficacy of ATO but also possibly reduce its dosages [4]. In this regard, using HL-60 cells which are reported to show resistance to arsenite, we found that delphinidin showed selective cytotoxic effects on the cells, but minimal effects on PBMNC, and sensitized the cells to arsenite, resulting in the enhancement of arsenite cytotoxicity (Yuan et al. manuscript in preparation). Therefore, our experimental data suggest that sensitization of HL-60 cells to arsenite achieved by the combination with delphinidin could benefit a reduced dosages of arsenite in clinical application, contributing to minimize side effect. The clinical trial planning is now underway.

Of note, it is well known that oxidative stress is involved in the mechanisms underlying the therapeutic efficacy of arsenite and plays a major role in the toxicity of arsenite $[4,13$, 77]. In fact, in order to maximally exploit effective ROS-mediated cell death without causing significant toxicity to normal cells, redox-based drug combination strategies have been proposed [3, 11, 18]. Based on the strategies, ROS-generating reagents including natural products derived substances, especially phenolic compounds, have received much attention due to their cytocidal effects on tumor cells but little on normal cells. In this regard, like the cytocidal effects of cyanidin-3-rutinoside on leukemia cells [10], delphinidin 3-sambubioside has also been demonstrated to induce apoptosis in HL-60 through ROS-mediated mitochondria pathway [78], suggesting these anthocyanins are good candidates for ROS-generating reagents, thereby possibly potentiate the action of 
ATO. Indeed, quercetin and/or genistein are flavonoid with multiple biochemical effects such as downregulation of phosphoinositide 3-kinase/Akt signaling pathway and NF- $\mathrm{B}$ transcription factor activity [79, 80], tyrosine kinase inhibition [81, 82]. Furthermore, both quercetin and genistein have been reported to selectively potentiate ATO-induced apoptosis via ROS generation resulted from intracellular GSH depletion, and activation of intrinsic and extrinsic apoptotic pathway in human leukemia cell lines such as HL-60, U937 and THP-1, but not in phytohemagglutinin-stimulated non-tumor peripheral blood lymphocytes [24, 83]. These results thus suggest that these flavonoids might be used to increase the clinical efficacy of ATO. Similar to flavonoids, subcytotoxic concentrations of curcumin also has been demonstrated to stimulate ROS production and potentiate apoptosis induction by ATO in leukemia cell lines via deregulation of Akt phosphorylation [25].

With these considerations in mind, a lot of preclinical and clinical trials have been carried out. For instance, we recently reported for the first time that 5-FU in combination with Vitex achieved an enhanced cytocidal effect on COLO 201 cells, but lesser cytotoxic effect on human PBMNC [9]. It has also been demonstrated that delphinidin induces apoptosis and inhibited NF- $\kappa B$ signaling in prostate tumor cells in vitro and in a human prostate tumor xenograft in nude mice in vivo [39]. Furthermore, twenty-five colon cancer patients without receiving prior therapy and surgery consumed $60 \mathrm{~g} /$ day $(20 \mathrm{~g} / 3 \mathrm{x} /$ day $)$ of black raspberry powder daily for $2-4$ weeks. Biopsies of normal-appearing and tumor tissues were taken from these patients before and after berry treatment. Intake of berries reduced proliferation rates and increased apoptosis in colon tumors but not in normal-appearing crypts [8]. On the other hand, supplementation of anthocyanins in the diet of cancer patients receiving chemotherapy did not result in increased inhibition of tumor development when compared to chemotherapy alone [84]. These conflicting findings suggest that a large scale of clinical trial is needed.

A phase I clinical trial has shown that quercetin, another one of flavonoids, can be safely administered to patients with ovarian cancer or hepatoma by intravenous injection of bolus at a dose of $1400 \mathrm{mg} / \mathrm{m}^{2}$ [82]. Moreover, the evidence of antitumor activity was seen in the clinical trial based on sustained fall in serum CA 125 levels, which is proposed for the use as a surrogate marker of response [85]. Furthermore, similar to our previous report [9], CDF (a difluorinated analog of curcumin), alone or in combination with 5-FU and oxaliplatin, was more potent than curcumin alone in reducing the number of chemoresistant HCT-116 and HT-29 colon cancer cells expressing CD44 and CD166 stem cell-like markers [56]. Concomitantly, cell growth inhibition, apoptosis induction and disintegration of colonospheres in these colon cancer cells were also observed in the study [56]. Moreover, clinical trials have confirmed the safety and feasibility to use curcumin in combination therapy with current chemotherapeutic treatments [7]. More recent results from a phaseI/II study on 21 advanced pancreatic cancer patients with gemcitabine-based chemotherapy have indicated that the median overall survival time of the patients after a treatment with curcumin plus gemcitabine or gemcitabine/S-1 combination was 161 days and 1-year survival rate of $19 \%$ (95\% confidence interval) [86]. Among eighteen evaluable patients, no 
patients experienced a partial or complete response and five patients (28\%) demonstrated stable disease according to Response Evaluation Criteria in Solid Tumors (RECIST) [86].

Although many encouraging results of in vitro and in vivo studies suggest polyphenolic compounds as a promising candidate for cancer therapy, either alone or in combination with current anticancer drugs, the therapeutic applications of these compounds in humans are limited by their high metabolic instability as well as poor absorption and bioavailability $[1,7,8,20,87]$. In this regard, the selective delivery of nanotechnology-based formulations of these polyphenolic compounds to tumors, alone or in combination with other anticancer drugs, has been of great interest [7, 26, 88]. For instance, pegylated liposomal quercetin was shown to significantly improve its solubility and bioavailability and be a potential application in the treatment of tumor based on a study using CT26 (a mouse colorectal carcinoma cell line), LL/2 (Lewis lung cancer cell line) and H22 (a hepatoma cell line) xenograft mice [88]. Furthermore, diverse curcumin formulations have been developed with different nanotechnology consisting of its encapsulation or conjugation with nanoparticles, polymeric micelles or liposomes to improve its stability, bioavailability and specific and sustained delivery into cancer cells and, consequently, its anticarcinogenic effects [7]. In particular, the systemic administration of gemcitabine plus polymeric micelle-encapsulated curcumin formulation enhanced greater bioavailability in plasma and tissues as compared to that of free curcumin in xenograft models of human pancreatic cancer established in athymic mice [89]. In consequence, the combinatory administration efficiently block tumor growth and metastases in this animal model of pancreatic cancer. Furthermore, an inhibition of NF- $\kappa \mathrm{B}$ and its targeted genes are implicated in the tumor growth inhibition [89]. Therefore, the use of nanotechnology-based formulations of polyphenolic compounds and their novel chemical analogs probably represents a potential alternative strategy of great clinical interest for overcoming the high metabolic instability and poor bioavailability of these compounds, which are among the principal factors limiting their therapeutic applications.

\section{Conclusion}

A striking global research on substances derived from natural products including polyphenolic compounds is being explored to understand the detailed mechanisms of their chemopreventive, antitumoral and chemosensibilizing activities against various types of aggressive and recurrent cancers. Besides the involvement of altered redox status in apoptosis induction triggered by these compounds, anti-inflammatory effects, antiangiogenesis, anti-invasiveness and induction of differentiation are well known to be implicated in their broad biological functions. It is worthy of note that flavonoids have been revealed to inhibit the function of ATP-binding cassette transporters such as multidrug resistance-associated proteins as well as P-glycoprotein [90], similar to our recent study [91]. On the other hand, a recent study demonstrated that berry anthocyanins, such as cyanidin-3-galactosidee, and cyanidin-3-glucoside as well as peonidin-3-glucosid, exhibit affinities for the efflux transporters breast cancer resistance protein (BCRP) and consequently may be actively transported out of intestinal tissues and endothelia [92]. However, the same report also demonstrated that some berry anthocyanins and 
anthocyanidins, such as delphinidin, cyanidin and cyanidin-3-rutinoside, act as BCRP inhibitor, while some of them, such as malvidin, malvidin-3-galactoside and petunidin, exhibited bimodal activities serving as BCRP substrates and inhibitors at low concentrations and high concentrations, respectively [92]. These findings suggest that a variety of biological activities of anthocyanins and anthocyanidins may be attributed in part to their inhibitory effects on those drug transporters, paradoxically, may be abolished as a result of efflux through those transporters. These findings also raised a pharmacological and pharmaceutical concern about formulatability of the dietary constituent, and warn us against the casual use of herbs and/or other botanicals in cancer patient care.

\section{Author details}

Bo Yuan, Masahiko Imai, Hidetomo Kikuchi, Shin Fukushima, Shingo Hazama, Takenori Akaike, Yuta Yoshino, Kunio Ohyama and Hiroo Toyoda Department of Clinical Molecular Genetics, School of Pharmacy, Tokyo University of Pharmacy $\mathcal{E}$ Life Sciences, Hachioji, Tokyo, Japan

Xiaomei $\mathrm{Hu}$

National Therapeutic Center of Hematology of Traditional Chinese Medicine, XiYuan Hospital, China Academy of Traditional Chinese Medicine, Beijing, P.R. China

Xiaohua Pei

The Third Affiliated Hospital of Beijing University of Traditional Chinese Medicine, Beijing, P.R. China

\section{Acknowledgement}

This work was supported in part by grants from Japan China Medical Association to B.Y. This work was also supported in part by grants from the Ministry of Education, Culture, Sports, Science and Technology and by the Promotion and Mutual Aid Corporation for Private Schools of Japan. The authors thank Dr. Chieko Hirobe for encouraging suggestions and arranging sample supply, and Dr. Yamato Kikkawa for providing HCC cell lines for this study.

\section{References}

[1] Fimognari C, Lenzi M, Hrelia P (2008) Chemoprevention of cancer by isothiocyanates and anthocyanins: Mechanisms of action and structure-activity relationship. Curr. Med. Chem. 15:440-447.

[2] Reddy L, Odhav B, Bhoola KD (2003) Natural products for cancer prevention: A global perspective. Pharmacol. Ther. 99:1-13.

[3] Trachootham D, Alexandre J, Huang P (2009) Targeting cancer cells by ros-mediated mechanisms: A radical therapeutic approach? Nat. Rev. Drug Discov. 8:579-591. 
[4] Yuan B, Yoshino Y, Kaise T, Toyoda H (2011) Application of arsenic trioxide therapy for patients with leukaemia. In: Sun HZ, editor. Biological Chemistry of As, Sb and Bi. John Wiley \& Sons, New York, pp.263-292.

[5] Meyerhardt JA, Mayer RJ (2005) Systemic therapy for colorectal cancer. N. Engl. J. Med. 352:476-487.

[6] Hurwitz H, Fehrenbacher L, Novotny W, Cartwright T, Hainsworth J, Heim W, Berlin J, Baron A, Griffing S, Holmgren E, Ferrara N, Fyfe G, Rogers B, Ross R, Kabbinavar F (2004) Bevacizumab plus irinotecan, fluorouracil, and leucovorin for metastatic colorectal cancer. N. Engl. J. Med. 350:2335-2342.

[7] Mimeault M, Batra SK (2011) Potential applications of curcumin and its novel synthetic analogs and nanotechnology-based formulations in cancer prevention and therapy. Chin. Med. 6:31.

[8] Wang LS, Stoner GD (2008) Anthocyanins and their role in cancer prevention. Cancer Lett. 269:281-290.

[9] Imai M, Kikuchi H, Yuan B, Aihara Y, Mizokuchi A, Ohyama K, Hirobe C, Toyoda H (2011) Enhanced growth inhibitory effect of 5-fluorouracil in combination with vitex agnus-castus fruits extract against a human colon adenocarcinoma cell line, colo 201. J. Chin. Clin. Med. 6:14-19.

[10] Feng R, Ni HM, Wang SY, Tourkova IL, Shurin MR, Harada H, Yin XM (2007) Cyanidin-3-rutinoside, a natural polyphenol antioxidant, selectively kills leukemic cells by induction of oxidative stress. J. Biol. Chem. 282:13468-13476.

[11] Zhang Y, Du Y, Le W, Wang K, Kieffer N, Zhang J (2011) Redox control of the survival of healthy and diseased cells. Antioxid. Redox Signal. 15:2867-2908.

[12] Indran IR, Tufo G, Pervaiz S, Brenner C (2011) Recent advances in apoptosis, mitochondria and drug resistance in cancer cells. Biochim. Biophys. Acta. 1807:735-745.

[13] Wang ZY, Chen Z (2008) Acute promyelocytic leukemia: From highly fatal to highly curable. Blood 111:2505-2515.

[14] Wei MC, Zong WX, Cheng EH, Lindsten T, Panoutsakopoulou V, Ross AJ, Roth KA, MacGregor GR, Thompson CB, Korsmeyer SJ (2001) Proapoptotic bax and bak: A requisite gateway to mitochondrial dysfunction and death. Science 292:727-730.

[15] Shimizu S, Narita M, Tsujimoto Y (1999) Bcl-2 family proteins regulate the release of apoptogenic cytochrome $\mathrm{c}$ by the mitochondrial channel vdac. Nature 399:483-487.

[16] Earnshaw WC, Martins LM, Kaufmann SH: Mammalian caspases (1999) Structure, activation, substrates, and functions during apoptosis. Annu. Rev. Biochem. 68:383-424.

[17] McCarthy NJ, Evan GI (1998) Methods for detecting and quantifying apoptosis. Curr. Top. Dev. Biol. 36:259-278.

[18] Schumacker PT (2006) Reactive oxygen species in cancer cells: Live by the sword, die by the sword. Cancer cell 10:175-176.

[19] Trachootham D, Zhou Y, Zhang H, Demizu Y, Chen Z, Pelicano H, Chiao PJ, Achanta G, Arlinghaus RB, Liu J, Huang P (2006) Selective killing of oncogenically transformed cells through a ros-mediated mechanism by beta-phenylethyl isothiocyanate. Cancer cell 10:241-252. 
[20] Cooke D, Steward WP, Gescher AJ, Marczylo T (2005) Anthocyans from fruits and vegetables--does bright colour signal cancer chemopreventive activity? Eur. J. Cancer 41:1931-1940.

[21] Imai M, Yuan B, Kikuchi H, Saito M, Ohyama K, Hirobe C, Oshima T, Hosoya T, Morita H, Toyoda H (2012) Growth inhibition of a human colon carcinoma cell, colo 201, by a natural product, vitex agnus-castus fruits extract, in vivo and in vitro. Adv, Biol. Chem. 2:20-28.

[22] Ohyama K, Akaike T, Imai M, Toyoda H, Hirobe C, Bessho T (2005) Human gastric signet ring carcinoma (kato-iii) cell apoptosis induced by vitex agnus-castus fruit extract through intracellular oxidative stress. Int. J. Biochem. Cell Biol. 37:1496-1510.

[23] Cvorovic J, Tramer F, Granzotto M, Candussio L, Decorti G, Passamonti S (2010) Oxidative stress-based cytotoxicity of delphinidin and cyanidin in colon cancer cells. Arch. Biochem. Biophys. 501:151-157.

[24] Ramos AM, Aller P (2008) Quercetin decreases intracellular gsh content and potentiates the apoptotic action of the antileukemic drug arsenic trioxide in human leukemia cell lines. Biochem. Pharmacol. 75:1912-1923.

[25] Sanchez Y, Simon GP, Calvino E, de Blas E, Aller P (2010) Curcumin stimulates reactive oxygen species production and potentiates apoptosis induction by the antitumor drugs arsenic trioxide and lonidamine in human myeloid leukemia cell lines. J. Pharmacol. Exp. Ther. 335:114-123.

[26] Singh M, Bhatnagar P, Srivastava AK, Kumar P, Shukla Y, Gupta KC (2011) Enhancement of cancer chemosensitization potential of cisplatin by tea polyphenols poly(lactide-co-glycolide) nanoparticles. J. Biomed. Nanotechnol. 7:202.

[27] Hertog MG, Hollman PC, Katan MB, Kromhout D (1993) Intake of potentially anticarcinogenic flavonoids and their determinants in adults in the netherlands. Nutr. Cancer 20:21-29.

[28] Zhang Y, Vareed SK, Nair MG (2005) Human tumor cell growth inhibition by nontoxic anthocyanidins, the pigments in fruits and vegetables. Life Sci. 76:1465-1472.

[29] Taylor PR, Greenwald P (2005) Nutritional interventions in cancer prevention. J. Clin. Oncol. 23:333-345.

[30] Berger D, Schaffner W, Schrader E, Meier B, Brattstrom A (2000) Efficacy of vitex agnus castus 1 . Extract ze 440 in patients with pre-menstrual syndrome (pms). Arch. Gynecol. Obstet. 264:150-153.

[31] Schellenberg R (2001) Treatment for the premenstrual syndrome with agnus castus fruit extract: Prospective, randomised, placebo controlled study. BMJ 322:134-137.

[32] Hirobe C, Qiao ZS, Takeya K, Itokawa H (1997) Cytotoxic flavonoids from vitex agnuscastus. Phytochemistry 46:521-524.

[33] Itokawa H, Qiao ZS, Hirobe C, Takeya K (1995) Cytotoxic limonoids and tetranortriterpenoids from melia azedarach. Chem. Pharm. Bull. (Tokyo) 43:1171-1175.

[34] Aggarwal BB, Sundaram C, Malani N, Ichikawa H (2007) Curcumin: The indian solid gold. Adv. Exp. Med. Biol. 595:1-75.

[35] Itokawa H, Shi Q, Akiyama T, Morris-Natschke SL, Lee KH (2008) Recent advances in the investigation of curcuminoids. Chin. Med. 3:11. 
[36] Seeram NP, Adams LS, Zhang Y, Lee R, Sand D, Scheuller HS, Heber D (2006) Blackberry, black raspberry, blueberry, cranberry, red raspberry, and strawberry extracts inhibit growth and stimulate apoptosis of human cancer cells in vitro. J. Agri. Food Chem. 54:9329-9339.

[37] Afaq F, Zaman N, Khan N, Syed DN, Sarfaraz S, Zaid MA, Mukhtar H (2008) Inhibition of epidermal growth factor receptor signaling pathway by delphinidin, an anthocyanidin in pigmented fruits and vegetables. Int. J. Cancer 123:1508-1515.

[38] Chen PN, Chu SC, Chiou HL, Chiang CL, Yang SF, Hsieh YS (2005) Cyanidin 3glucoside and peonidin 3-glucoside inhibit tumor cell growth and induce apoptosis in vitro and suppress tumor growth in vivo. Nutr. Cancer 53:232-243.

[39] Hafeez BB, Siddiqui IA, Asim M, Malik A, Afaq F, Adhami VM, Saleem M, Din M, Mukhtar H (2008) A dietary anthocyanidin delphinidin induces apoptosis of human prostate cancer pc3 cells in vitro and in vivo: Involvement of nuclear factor-kappab signaling. Cancer Res. 68:8564-8572.

[40] Reddivari L, Vanamala J, Chintharlapalli S, Safe SH, Miller JC, Jr. (2007) Anthocyanin fraction from potato extracts is cytotoxic to prostate cancer cells through activation of caspase-dependent and caspase-independent pathways. Carcinogenesis 28:2227-2235.

[41] Chang YC, Huang HP, Hsu JD, Yang SF, Wang CJ (2005) Hibiscus anthocyanins rich extract-induced apoptotic cell death in human promyelocytic leukemia cells. Toxicol. Appl. Pharmacol. 205:201-212.

[42] Fanciulli M, Bruno T, Giovannelli A, Gentile FP, Di Padova M, Rubiu O, Floridi A (2000) Energy metabolism of human lovo colon carcinoma cells: Correlation to drug resistance and influence of lonidamine. Clin. Cancer Res. 6:1590-1597.

[43] Warburg O (1956) On respiratory impairment in cancer cells. Science 124:269-270.

[44] Kachadourian R, Day BJ (2006) Flavonoid-induced glutathione depletion: Potential implications for cancer treatment. Free Radic. Biol. Med. 41:65-76.

[45] Elliott AJ, Scheiber SA, Thomas C, Pardini RS (1992) Inhibition of glutathione reductase by flavonoids. A structure-activity study. Biochem. Pharmacol. 44:1603-1608.

[46] Dickancaite E, Nemeikaite A, Kalvelyte A, Cenas N (1998) Prooxidant character of flavonoid cytotoxicity: Structure-activity relationships. Biochem. Mol. Biol. Int. 45:923930.

[47] Sergediene E, Jonsson K, Szymusiak H, Tyrakowska B, Rietjens IM, Cenas N (1999) Prooxidant toxicity of polyphenolic antioxidants to hl-60 cells: Description of quantitative structure-activity relationships. FEBS Lett. 462:392-396.

[48] Hou DX, Ose T, Lin S, Harazoro K, Imamura I, Kubo M, Uto T, Terahara N, Yoshimoto M, Fujii M (2003) Anthocyanidins induce apoptosis in human promyelocytic leukemia cells: Structure-activity relationship and mechanisms involved. Int. J. Oncol. 23:705-712.

[49] Hou DX, Kai K, Li JJ, Lin S, Terahara N, Wakamatsu M, Fujii M, Young MR, Colburn N (2004) Anthocyanidins inhibit activator protein 1 activity and cell transformation: Structure-activity relationship and molecular mechanisms. Carcinogenesis 25:29-36.

[50] Hou DX, Yanagita T, Uto T, Masuzaki S, Fujii M (2005) Anthocyanidins inhibit cyclooxygenase-2 expression in lps-evoked macrophages: Structure-activity relationship and molecular mechanisms involved. Biochem. Pharmacol. 70:417-425. 
[51] Zhang H, Berezov A, Wang Q, Zhang G, Drebin J, Murali R, Greene MI (2007) Erbb receptors: From oncogenes to targeted cancer therapies. J. Clin. Invest. 117:2051-2058.

[52] Ohyama K, Akaike T, Hirobe C, Yamakawa T (2003) Cytotoxicity and apoptotic inducibility of vitex agnus-castus fruit extract in cultured human normal and cancer cells and effect on growth. Biol. Pharm. Bull. 26:10-18.

[53] Imai M, Kikuchi H, Denda T, Ohyama K, Hirobe C, Toyoda H (2009) Cytotoxic effects of flavonoids against a human colon cancer derived cell line, COLO 201: A potential natural anti-cancer substance. Cancer Lett. 276:74-80.

[54] Zhou Y, Liu YE, Cao J, Zeng G, Shen C, Li Y, Zhou M, Chen Y, Pu W, Potters L, Shi YE (2009) Vitexins, nature-derived lignan compounds, induce apoptosis and suppress tumor growth. Clin. Cancer Res. 15:5161-5169.

[55] Epelbaum R, Schaffer M, Vizel B, Badmaev V, Bar-Sela G (2010) Curcumin and gemcitabine in patients with advanced pancreatic cancer. Nutr. Cancer 62:1137-1141.

[56] Kanwar SS, Yu Y, Nautiyal J, Patel BB, Padhye S, Sarkar FH, Majumdar AP (2011) Difluorinated-curcumin (cdf): A novel curcumin analog is a potent inhibitor of colon cancer stem-like cells. Pharm. Res. 28:827-838.

[57] Reuter S, Eifes S, Dicato M, Aggarwal BB, Diederich M (2008) Modulation of antiapoptotic and survival pathways by curcumin as a strategy to induce apoptosis in cancer cells. Biochem. Pharmacol. 76:1340-1351.

[58] Yoshino M, Haneda M, Naruse M, Htay HH, Tsubouchi R, Qiao SL, Li WH, Murakami K, Yokochi T (2004) Prooxidant activity of curcumin: Copper-dependent formation of 8hydroxy-2'-deoxyguanosine in DNA and induction of apoptotic cell death. Toxico. in vitro 18:783-789.

[59] Kuo ML, Huang TS, Lin JK (1996) Curcumin, an antioxidant and anti-tumor promoter, induces apoptosis in human leukemia cells. Biochim. Biophys. Acta. 1317:95-100.

[60] Kim MS, Kang HJ, Moon A (2001) Inhibition of invasion and induction of apoptosis by curcumin in h-ras-transformed mcf10a human breast epithelial cells. Arch. Pharm. Res. 24:349-354.

[61] Syng-Ai C, Kumari AL, Khar A (2004) Effect of curcumin on normal and tumor cells: Role of glutathione and bcl-2. Mol. Cancer Ther. 3:1101-1108.

[62] Wahlstrom B, Blennow G (1978) A study on the fate of curcumin in the rat. Acta. Pharmacol. Toxicol. (Copenh) 43:86-92.

[63] Anand P, Kunnumakkara AB, Newman RA, Aggarwal BB (2007) Bioavailability of curcumin: Problems and promises. Mol. Pharm. 4:807-818.

[64] Padhye S, Banerjee S, Chavan D, Pandye S, Swamy KV, Ali S, Li J, Dou QP, Sarkar FH (2009) Fluorocurcumins as cyclooxygenase-2 inhibitor: Molecular docking, pharmacokinetics and tissue distribution in mice. Pharm. Res. 26:2438-2445.

[65] Padhye S, Yang H, Jamadar A, Cui QC, Chavan D, Dominiak K, McKinney J, Banerjee S, Dou QP, Sarkar FH (2009) New difluoro knoevenagel condensates of curcumin, their schiff bases and copper complexes as proteasome inhibitors and apoptosis inducers in cancer cells. Pharm. Res. 26:1874-1880. 
[66] Yoshino Y, Yuan B, Miyashita SI, Iriyama N, Horikoshi A, Shikino O, Toyoda H, Kaise T (2009) Speciation of arsenic trioxide metabolites in blood cells and plasma of a patient with acute promyelocytic leukemia. Anal. Bioanal. Chem. 393:689-697.

[67] Kiguchi T, Yoshino Y, Yuan B, Yoshizawa S, Kitahara T, Akahane D, Gotoh M, Kaise T, Toyoda H, Ohyashiki K (2010) Speciation of arsenic trioxide penetrates into cerebrospinal fluid in patients with acute promyelocytic leukemia. Leuk. Res. 34:403405.

[68] Chen GQ, Shi XG, Tang W, Xiong SM, Zhu J, Cai X, Han ZG, Ni JH, Shi GY, Jia PM, Liu MM, He KL, Niu C, Ma J, Zhang P, Zhang TD, Paul P, Naoe T, Kitamura K, Miller W, Waxman S, Wang ZY, de The H, Chen SJ, Chen Z (1997) Use of arsenic trioxide (As2O3) in the treatment of acute promyelocytic leukemia (APL): I. As2O3 exerts dosedependent dual effects on apl cells. Blood 89:3345-3353.

[69] Soignet SL, Maslak P, Wang ZG, Jhanwar S, Calleja E, Dardashti LJ, Corso D, DeBlasio A, Gabrilove J, Scheinberg DA, Pandolfi PP, Warrell RP, Jr. (1998) Complete remission after treatment of acute promyelocytic leukemia with arsenic trioxide. N. Engl. J. Med. 339:1341-1348.

[70] Iriyama N, Yoshino Y, Yuan B, Horikoshi A, Hirabayashi Y, Hatta Y, Toyoda H, Takeuchi J (2012) Speciation of arsenic trioxide metabolites in peripheral blood and bone marrow from an acute promyelocytic leukemia patient. J. Hematol. Oncol. 5:1.

[71] Yuan B, Ohyama K, Bessho T, Toyoda H (2006) Contribution of inducible nitric oxide synthase and cyclooxygenase-2 to apoptosis induction in smooth chorion trophoblast cells of human fetal membrane tissues. Biochem. Biophys. Res. Commun. 341:822-827.

[72] Yuan B, Ohyama K, Bessho T, Uchide N, Toyoda H (2008) Imbalance between ros production and elimination results in apoptosis induction in primary smooth chorion trophoblast cells prepared from human fetal membrane tissues. Life Sci. 82:623-630.

[73] Yuan B, Ohyama K, Takeichi M, Toyoda H (2009) Direct contribution of inducible nitric oxide synthase expression to apoptosis induction in primary smooth chorion trophoblast cells of human fetal membrane tissues. Int. J. Biochem. Cell Biol. 41:10621069.

[74] Yoshino Y, Yuan B, Kaise T, Takeichi M, Tanaka S, Hirano T, Kroetz DL, Toyoda H (2011) Contribution of aquaporin 9 and multidrug resistance-associated protein 2 to differential sensitivity to arsenite between primary cultured chorion and amnion cells prepared from human fetal membranes. Toxicol. Appl. Pharmacol. 257:198-208.

[75] Dilda PJ, Hogg PJ (2007) Arsenical-based cancer drugs. Cancer Treat Rev. 33:542-564.

[76] Litzow MR (2008) Arsenic trioxide. Expert Opin. Pharmacother. 9:1773-1785.

[77] Ninomiya M, Kajiguchi T, Yamamoto K, Kinoshita T, Emi N, Naoe T (2006) Increased oxidative DNA products in patients with acute promyelocytic leukemia during arsenic therapy. Haematologica 91:1571-1572.

[78] Hou DX, Tong X, Terahara N, Luo D, Fujii M (2005) Delphinidin 3-sambubioside, a hibiscus anthocyanin, induces apoptosis in human leukemia cells through reactive oxygen species-mediated mitochondrial pathway. Arch. Biochem. Biophys. 440:101-109. 
[79] Sarkar FH, Adsule S, Padhye S, Kulkarni S, Li Y (2006) The role of genistein and synthetic derivatives of isoflavone in cancer prevention and therapy. Mini. Rev. Med. Chem. 6:401-407.

[80] Sharma H, Sen S, Singh N (2005) Molecular pathways in the chemosensitization of cisplatin by quercetin in human head and neck cancer. Cancer Biol. Ther. 4:949-955.

[81] Akiyama T, Ishida J, Nakagawa S, Ogawara H, Watanabe S, Itoh N, Shibuya M, Fukami Y (1987) Genistein, a specific inhibitor of tyrosine-specific protein kinases. J. Biol. Chem. 262:5592-5595.

[82] Ferry DR, Smith A, Malkhandi J, Fyfe DW, deTakats PG, Anderson D, Baker J, Kerr DJ (1996) Phase I clinical trial of the flavonoid quercetin: Pharmacokinetics and evidence for in vivo tyrosine kinase inhibition. Clin. Cancer Res. 2:659-668.

[83] Sanchez Y, Amran D, Fernandez C, de Blas E, Aller P (2008) Genistein selectively potentiates arsenic trioxide-induced apoptosis in human leukemia cells via reactive oxygen species generation and activation of reactive oxygen species-inducible protein kinases (p38-MAPK, AMPK). Int. J. Cancer 123:1205-1214.

[84] Bode U, Hasan C, Hulsmann B, Fleischhack G (1999) Recancostat compositum therapy does not prevent tumor progression in young cancer patients. Klin. Padiatr. 211:353355.

[85] Rustin GJ, van der Burg ME, Berek JS (1993) Advanced ovarian cancer. Tumour markers. Ann. Oncol. 4 Suppl 4:71-77.

[86] Kanai M, Yoshimura K, Asada M, Imaizumi A, Suzuki C, Matsumoto S, Nishimura T, Mori Y, Masui T, Kawaguchi Y, Yanagihara K, Yazumi S, Chiba T, Guha S, Aggarwal BB (2011) A phase I/II study of gemcitabine-based chemotherapy plus curcumin for patients with gemcitabine-resistant pancreatic cancer. Cancer Chemother. Pharmacol. 68:157-164.

[87] Thomasset S, Teller N, Cai H, Marko D, Berry DP, Steward WP, Gescher AJ (2009) Do anthocyanins and anthocyanidins, cancer chemopreventive pigments in the diet, merit development as potential drugs? Cancer Chemother. Pharmacol. 64:201-211.

[88] Yuan ZP, Chen LJ, Fan LY, Tang MH, Yang GL, Yang HS, Du XB, Wang GQ, Yao WX, Zhao QM, Ye B, Wang R, Diao P, Zhang W, Wu HB, Zhao X, Wei YQ (2006) Liposomal quercetin efficiently suppresses growth of solid tumors in murine models. Clin. Cancer Res. 12:3193-3199.

[89] Bisht S, Mizuma M, Feldmann G, Ottenhof NA, Hong SM, Pramanik D, Chenna V, Karikari C, Sharma R, Goggins MG, Rudek MA, Ravi R, Maitra A, Maitra A (2010) Systemic administration of polymeric nanoparticle-encapsulated curcumin (nanocurc) blocks tumor growth and metastases in preclinical models of pancreatic cancer. Mol. Cancer Ther. 9:2255-2264.

[90] Kitagawa S (2006) Inhibitory effects of polyphenols on p-glycoprotein-mediated transport. Biol. Pharm. Bull. 29:1-6.

[91] Ishii K, Tanaka S, Kagami K, Henmi K, Toyoda H, Kaise T, Hirano T (2010) Effects of naturally occurring polymethyoxyflavonoids on cell growth, p-glycoprotein function, cell cycle, and apoptosis of daunorubicin-resistant $\mathrm{T}$ lymphoblastoid leukemia cells. Cancer Invest. 28:220-229. 
[92] Dreiseitel A, Oosterhuis B, Vukman KV, Schreier P, Oehme A, Locher S, Hajak G, Sand PG (2009) Berry anthocyanins and anthocyanidins exhibit distinct affinities for the efflux transporters bcrp and mdr1. Br. J. Pharmacol. 158:1942-1950. 